\title{
Primeras observaciones de Libelloides longicornis (Scopoli, 1763) (Neuroptera: Ascalaphidae) en Gipuzkoa.
}

\section{First observations of Libelloides longicornis (Scopoli, 1763) (Neuroptera: Ascalaphidae) in Gipuzkoa.}

Iker Novoa-Fariñas ${ }^{1,2}$, Egoitz Alkorta ${ }^{3}$, Beñat Lardizabal ${ }^{4}$

*

\section{Resumen}

Se describen las primeras observaciones de Libelloides longicornis en la provincia de Gipuzkoa.

Palabras clave: Ascalaphidae, Libelloides, longicornis, Oñati, Gipuzkoa, Aizkorri-Aratz.

\section{Abstract}

Description of the first observations of Libelloides longicornis in the province of Gipuzkoa. Key words: Ascalaphidae, owlflies, Libelloides, longicornis, Oñati, Gipuzkoa, AizkorriAratz.

\section{Laburpena}

Gipuzkoan Libelloides longicornis espeziearen lehenengo behaketaren datuak ematen dira.

Gako hitzak: Ascalaphidae, Libelloides, longicornis, Oñati, Gipuzkoa, Aizkorri-Aratz.

1 Oñatiko Naturzaliak 20560 Oñati

2 Asociación ZERYNTHIA Madre de Dios, $n^{\circ} 14-7^{\circ} \mathrm{D}, \mathrm{E}-26004$ Logroño

3 Sociedad de Ciencias Aranzadi Zientzia Elkartea Zorroagagaina 11. 20014 Donostia-San Sebastián

4 Lardizabal kalea 1, 3 esk., Segura.

*Correspondencia: ikernovo@hotmail.com 
Los ascaláfidos (Ascalaphidae) son una familia de insectos pertenecientes al orden de los neurópteros. Son hábiles voladores y depredadores de otros insectos. Su morfología es llamativa, con algunos rasgos que recuerdan superficialmente a otros órdenes de insectos como las libélulas (orden Odonata) y las mariposas (orden Lepidoptera). En la Península Ibérica se conocen 10 especies agrupadas en 4 géneros (Monserrat et al., 2014). En la Comunidad Autónoma Vasca está comprobada la presencia de una de esas especies, Libelloides longicornis, en Bizkaia y Araba. (Monserrat \& Triviño, 2013; Sesma et al., 2016). Sin embargo, no se disponía de registros previos de insectos de esta familia para la provincia de Gipuzkoa. En el presente trabajo se ofrece la primera cita provincial de Libelloides longicornis (Scopoli, 1763), localizado dentro del Parque Natural de Aizkorri-Aratz. La nomenclatura científica y autoría de los taxones botánicos mencionados sigue los criterios de Flora lbérica, y para los nombres comunes de las plantas se sigue la propuesta de Mateo (2016).

Libelloides longicornis es un libeluloide de actividad diurna, de distribución atlantomediterránea (Aspöck et al., 2001) y presente en muchas zonas de la península ibérica. Como otros ascaláfidos es un depredador, consumiendo otros insectos tanto de adulto como durante su fase larvaria. Los imagos de esta especie viven unas pocas semanas, pudiendo ser observados a finales de primavera y durante la época estival (Monserrat et al., 2012). En el País Vasco existen citas de su presencia en Bizkaia y Araba y la presente nota sirve para confirmar que ocupa los tres territorios históricos de la comunidad autónoma.

El 10.07.2018 durante un muestreo de mariposas realizado por los autores en Oñati, pudo ser observado un ascaláfido en vuelo en el paraje conocido como Kortakogain. Ya desde el primer momento se sospechó que pudiera ser Libelloides longicornis, pero al no verlo posado no fue posible determinar la especie con seguridad. Por ello, una semana después, el 17.07.2018 se repitió la visita al mismo lugar con el objetivo de poder confirmar la observación. Esta vez pudieron observarse 2 ejemplares en el mismo lugar, un macho y una hembra (figura 1). Ambos se encontraban volando juntos y también posados. Se obtuvieron fotografías de los dos. Posteriormente, el 10.09.2018 se realizó una tercera visita al mismo lugar, pero ya no pudo detectarse la especie.

El hábitat que ocupa es una cantera caliza abandonada, con vegetación predominantemente arbustiva. Hay zonas herbosas donde hay rocas y poco suelo, así como vegetación ruderal en los caminos y lugares removidos. El estrato arbustivo lo forman principalmente enebros (Juniperus communis L.), zarzas (Rubus sp.), cornejos (Cornus sanguinea L.), tojos (Ulex europaeus L.) y sauces (Salix atrocinerea Brot.). Entre la vegetación herbosa destacan las viboreras (Echium vulgare L.), helicriso (Helichrysum stoechas (L.) Moench.), valeriana roja fina (Centranthus lecoqii Jord.) e hinojo (Foeniculum vulgare Mill.). El enclave, situado en el paraje conocido como 
Kortakogain en el municipio de Oñati, está incluido en el Parque Natural y Zona de Especial Conservación de Aizkorri-Aratz. Está situado a una altitud de $600 \mathrm{~m}$ en la solana del monte Aloña, en las cuadrículas UTM de 1x1 km 30 TWN4760 y 30TWN4660.

Es muy probable que la especie se encuentre presente en más zonas de Gipuzkoa. La presente nota puede animar a otras personas a buscar éste y otros ascaláfidos en zonas propicias de la provincia, para tratar de mejorar el conocimiento que se tiene de este grupo.

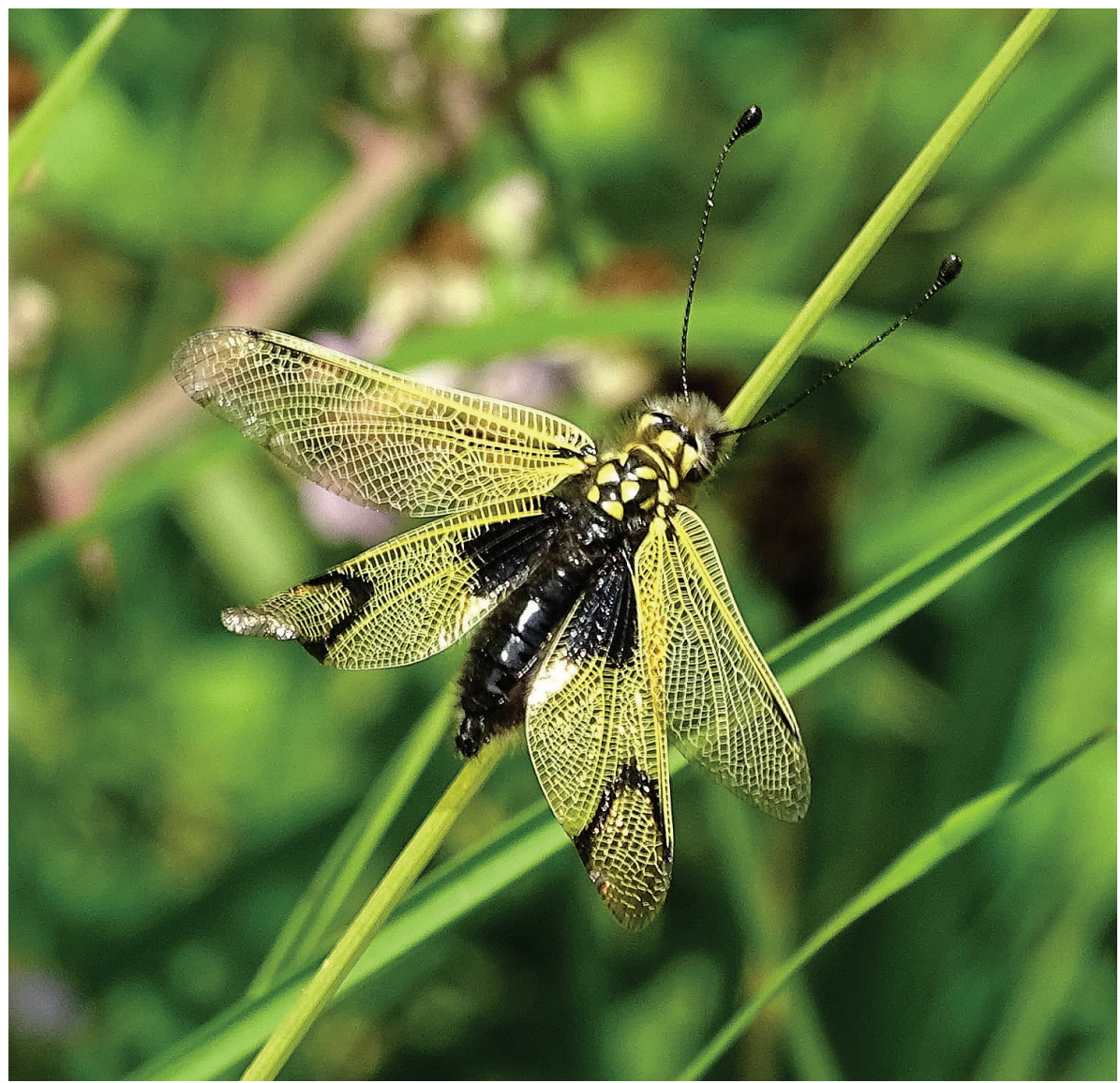

Fig. 1.- Ejemplar de Libelloides longicornis en Oñati 17.07.2018

Fig. 1.- Specimen of Libelloides longicornis in Oñati 17.07.2018 


\section{Agradecimientos}

A Haizea Agirre por la compañía en las jornadas de campo. A Pablo Sánchez Fernández y un revisor anónimo por sus valiosas aportaciones, que han contribuido a mejorar el texto original.

\section{Bibliografía}

Aspöck, H., Hölzel, H., Aspöck, U. 2001. Kommentierter Katalog der Neuropterida (Insecta: Raphidioptera, Megaloptera, Neuroptera) der Westpaläarktis. Denisia 2:1-606.

Castroviejo, S. (coord. gen.). 1986-2012. Flora ibérica. 1-8, 10-15, 17-18, 21. Real Jardín Botánico, CSIC, Madrid.

Mateo, G. 2016. Los nombres comunes de las plantas. Monografías de flora montiberica. 7.

Monserrat, V.J., Badano, D., Acevedo, F. 2014. Nuevos datos de ascaláfidos para la península Ibérica, con una nueva especie para la fauna europea (Insecta, Neuropterida, Neuroptera, Ascalaphidae). Heteropterus, Rev.Entomol. 14: 147-167.

Monserrat, V.J., Triviño, V., 2013. Atlas de los neurópteros de la Península Ibérica e Islas Baleares (Insecta, Neuroptera: Megaloptera, Raphidioptera, Planipennia) / Atlas of the Iberian and Balearic lacewings (Insecta, Neuroptera: Megaloptera, Raphidioptera, Planipennia). Monografías de la Sociedad Entomológica Aragonesa vol. 13, Zaragoza.

Monserrat, V.J., Acevedo, F., Triviño, V. 2012. Los ascaláfidos de la Península Ibérica y Baleares (Insecta: Neuroptera: Ascalaphidae). Heteropterus, Rev. Entomol. 12(1): 33-58

Sesma, J.M., Cabellos, N., Noval, N., Pedreira. A. 2016. Género Libelloides. Clave de especies. Disponible en: www.biodiversidadvirtual.org/taxofoto/sites/default/files/neu.1_0.pdf. Consultado el 12.10.2018.

Fecha de recepcion/Date of reception: 19/12/2018

Fecha de aceptacion/Date of acceptance: 11/02/2019

Editor Asociado/Associate editor: Alberto Castro 